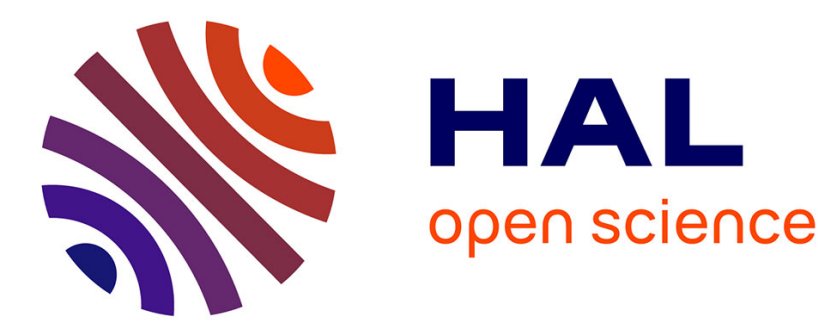

\title{
Une petite entreprise qui ne connaît pas la crise
}

\author{
Laurence Dumoulin, Séverine Germain, Anne-Cécile Douillet
}

\section{To cite this version:}

Laurence Dumoulin, Séverine Germain, Anne-Cécile Douillet. Une petite entreprise qui ne connaît pas la crise: Le succès de la vidéosurveillance au regard de la littérature internationale. Champ Pénal, 2010, VII, pp.Varia. halshs-00578476

\section{HAL Id: halshs-00578476 \\ https://shs.hal.science/halshs-00578476}

Submitted on 21 Mar 2011

HAL is a multi-disciplinary open access archive for the deposit and dissemination of scientific research documents, whether they are published or not. The documents may come from teaching and research institutions in France or abroad, or from public or private research centers.
L'archive ouverte pluridisciplinaire HAL, est destinée au dépôt et à la diffusion de documents scientifiques de niveau recherche, publiés ou non, émanant des établissements d'enseignement et de recherche français ou étrangers, des laboratoires publics ou privés. 


\title{
Champ pénal / Penal field, nouvelle revue internationale de criminologie
}

Vol. VII | 2010 :

Le traitement de l’immigration, entre logique administrative et logique pénale Articles ("Varia")

\section{Une petite entreprise qui ne connaît pas la crise}

\author{
Le succès de la vidéosurveillance au regard de la \\ littérature internationale
}

\author{
Laurence Dumoulin, Séverine Germain et Anne-Cécile Douillet
}

\section{Résumés}

Il est paradoxal de constater que la vidéosurveillance se diffuse dans de nombreux espaces sociaux et nationaux alors même que ses effets en termes de prévention et/ou de répression de la délinquance et de réassurance des populations ne sont pas démontrés. À travers une analyse critique de la littérature internationale sur la vidéosurveillance, l'article s'attache à identifier les différents schèmes explicatifs qui sont mis en avant pour comprendre la fortune de cette technologie. Trois types d'approche, qui constituent autant de façons de problématiser la portée sociale et politique de la vidéosurveillance, sont distingués : les surveillance studies, les études d'impact et les analyses d'usages. L'article discute ces travaux et les réponses qu'ils apportent à la compréhension du développement de la vidéosurveillance. Il souligne l'intérêt de travailler par analyse de cas, à l'échelle microsociologique, pour saisir avec finesse les mécanismes localement observables par lesquels de nouveaux acteurs sont enrôlés dans le dispositif et de nouvelles légitimations rendues possibles.

This article deals with a paradox: the spread of close-circuit television (CCTV) cameras in numerous social and national spaces whereas its effects on crime, in terms of crime prevention and $\backslash$ or law enforcement and community reassurance are not demonstrated. Through a critical analysis of the international literature on CCTV, this article attempts to identify the various reasons advanced to explain the 'success' of this technology. Three kinds of approach, which embody three ways of defining the political and social impact of CCTV, can be distinguished: surveillance studies, impact analyses and use studies. This paper discusses these works and the answers they bring to the understanding of CCTV development. It claims that micro-level case study analysis allows grasping with sharpness the locally observable mechanisms by which new actors can be enrolled in the device and 


\section{Entrées d'index}

Index de mots-clés : état de l'art, genèse, usages, légitimation, vidéosurveillance

Index by keyword : CCTV, state of the art, genesis, uses, legitimation

Index géographique : France, Royaume-Uni, Europe, États-Unis

Index chronologique : XXIe siècle

\section{Texte intégral}

\section{Introduction}

À partir des années 1980, les travaux sur la vidéosurveillance se sont multipliés et, dans la littérature de sciences sociales, le thème connaît un succès qui n'a pas été démenti depuis. Cette inflation académique va de pair avec le déploiement de dispositifs de vidéosurveillance ; ils concernent un nombre de plus en plus élevé de pays $^{1}$, se diversifient (espaces privés, espaces publics et ouverts au public...), bénéficient d'améliorations techniques et font l'objet d'entreprises de légitimation, comme en témoigne en France la substitution du terme « vidéoprotection » à celui de « vidéosurveillance $»^{2}$.

Au delà du simple constat du développement de la vidéosurveillance, la plupart des travaux cherchent à l'expliquer et à en analyser les effets, mobilisant pour ce faire trois types d'approche. Même si elles coexistent dans quelques rares enquêtes ${ }^{3}$, ces trois perspectives sont dans l'ensemble assez cloisonnées car elles constituent autant de façons de problématiser la portée sociale et politique de la vidéosurveillance. La première approche s'intéresse aux dangers potentiels ou avérés - c'est un des points de discussion - de la vidéosurveillance. Elle se développe essentiellement dans ce qui est maintenant identifié comme le champ des surveillance studies, pour lesquelles cette technologie est un des éléments d'une société de surveillance, c'est-à-dire de contrôle social accru. Un deuxième type de travaux vise à évaluer l'efficacité de la vidéosurveillance. Ces études d'impact ont fleuri à partir de la fin des années 1980, essentiellement dans le monde anglo-américain, et ont donné lieu à de vives controverses dans le champ scientifique. Les difficultés théoriques et pratiques ont fait évoluer les questions de recherche et ces études ont progressivement délaissé la question de l'impact de la vidéosurveillance sur le crime pour lui substituer une analyse des conditions de son efficacité. Avec ce changement de perspective, ce sont plus largement les effets empiriquement observables de la vidéosurveillance (sur un quartier mais aussi un secteur d'activité, des métiers...) qui sont ciblés. La vidéosurveillance est ainsi réintégrée dans son contexte de déploiement. Ce type d'études reste toutefois marqué par une visée normative au sens où il s'agit de cerner ce qui caractérise les situations d'échec de la vidéosurveillance ou de voir à quelles conditions celle-ci peut atteindre les buts qui lui ont été assignés. Le troisième type d'approche enfin s'intéresse aux mécanismes de genèse et de diffusion de la vidéosurveillance. $\mathrm{Si}$ des considérations générales sur les facteurs de son développement sont présentes de façon éparse dans bon nombre d'écrits, certains travaux s'attachent plus précisément à comprendre les processus sous-jacents au déploiement de dispositifs de vidéosurveillance particuliers. Les auteurs se concentrent davantage alors sur les usages pluriels, développés en situation par les opérateurs, et sur l'appropriation de l'objet par les différents acteurs qui, dans les juridictions, les polices fédérales, nationales ou locales, les centres commerciaux, 
traitent avec les technologies de vidéosurveillance. L'accent est mis sur les processus sociologiques qui permettent d'expliquer ce que l'on pourrait qualifier de succès de la vidéosurveillance, non pas sur le plan de son efficacité mais sur celui de sa généralisation comme outil de l'action publique en matière de sécurité.

Mise en évidence des dangers, évaluation des impacts et étude des usages : telles sont donc les principales problématiques de l'immense production de sciences sociales sur le thème de la vidéosurveillance. À travers tous ces travaux, un paradoxe se dessine : les dispositifs de vidéosurveillance se développent incontestablement depuis les années 1990 - au nom de la prévention de la délinquance et de la sécurité des citoyens - alors même que leur efficacité est loin d'être démontrée et que l'installation, l'exploitation et la maintenance de ces systèmes sont de plus en plus coûteuses, dans un contexte financier très tendu. Comment expliquer que la vidéosurveillance « ne connaisse pas la crise » ? Une fois discutée la thèse de l'efficacité des dispositifs de vidéosurveillance (1), restent pour répondre à cette question les propositions discutables défendues par les surveillance studies (2) et les réponses circonstanciées - mais plus convaincantes - des analyses sur les processus de développement de la vidéosurveillance (3).

\section{La vidéosurveillance comme outil de lutte contre la délinquance : efficacité et effets}

Face à la prolifération de dispositifs présentés par leurs promoteurs comme ayant des vertus dissuasives pour les délinquants et rassurantes pour les citoyens-usagers, de nombreux chercheurs ont entrepris d'identifier l'impact des dispositifs de vidéosurveillance sur l'évolution de la délinquance et le sentiment d'insécurité, notamment dans le monde anglo-américain (Grande-Bretagne, États-Unis et, dans une moindre mesure, Canada). D'abord menées dans une perspective globale (Does CCTV work?) (1), elles ont progressivement été réorientées vers une interrogation sur les conditions de fonctionnement de la vidéosurveillance (2). La plupart des auteurs s'inscrivant dans cette problématique de l'efficacité de la vidéosurveillance s'accordent aujourd'hui sur la nécessité de comprendre dans quelles circonstances elle peut agir sur la délinquance.

\section{La vidéosurveillance fonctionne-t-elle?}

Les études d'impact de la vidéosurveillance, bien qu'extrêmement nombreuses, ne permettent pas de conclure à son efficacité : alors qu'un certain nombre d'études suggèrent un effet positif (Tilley, 1993 ; Brown, 1995), d'autres parviennent au résultat opposé (Grandmaison, Tremblay, 1997). Une méta-analyse de 41 études d'impact (Welsh, Farrington, 2008), révèle que la vidéosurveillance réduit faiblement la délinquance mais que son efficacité est à nuancer en fonction des contextes écologiques et des types d'infractions considérés. La vidéosurveillance serait ainsi moins efficace dans les transports en commun ou les centres-villes que dans les parkings. Les diverses revues de littérature disponibles (Heilmann, Mornet, 2001 ; Le Goff, 2008) parviennent à des conclusions similaires.

Certaines études se sont plus spécifiquement intéressées aux effets sur le sentiment d'insécurité. Si plusieurs d'entre elles concluent à un impact de la vidéosurveillance (Chatterton, Frenz, 1994 ; Ditton, 2000), pour d'autres, la vidéosurveillance est sans effet sur le ressenti individuel (Webb, Laycock, 1992). À partir de douze enquêtes d'opinion réalisées dans le cadre d'une évaluation commanditée par le Home Office, 
Martin Gill et Angela Spriggs (2005)suggèrent que la crainte d'être victime est plus liée au niveau de délinquance qu'à la présence de caméras. D’une part, le sentiment d'insécurité a décliné dans les sept zones où la victimisation reportée a effectivement diminué. D'autre part, le fait de savoir qu'il y a des caméras ne réduit pas forcément le sentiment d'insécurité : les enquêtés conscients de la présence des caméras sont plus inquiets que ceux qui l'ignorent.

Au delà de la disparité des résultats obtenus et du manque potentiel d'objectivité des études réalisées ${ }^{4}$, ces travaux souffrent d'une fragilité intrinsèque. Celle-ci tient aux difficultés de réalisation de ces études. En effet, isoler, mesurer et évaluer les effets directs ou indirects de la vidéosurveillance sur la délinquance reste un pari méthodologique particulièrement difficile à relever ${ }^{5}$. Le premier écueil tient à la singularité de chaque programme de vidéosurveillance. En effet, chaque dispositif est unique (nombre et type de caméras, topographie du lieu surveillé et de la délinquance qui y est commise, objectifs assignés au départ, etc.), ce qui rend les comparaisons délicates. Le choix des zones-tests est lui aussi crucial. D’une part, il faut au préalable sélectionner des secteurs où le nombre de faits recensés est suffisamment important pour être traité statistiquement. D'autre part, il est indispensable de choisir une zone témoin (sans caméras) comparable à la zone-test pour contrôler l'impact des caméras sur cette dernière ${ }^{6}$. Le cadre temporel de l'étude doit également être défini avec attention et notamment inclure des mesures de la délinquance sur des durées suffisamment étendues pour repérer les tendances de longue période et les variations saisonnières. Il faut en outre non seulement mesurer la robustesse des effets de la vidéosurveillance mais également leur persistance dans le temps. Il a par exemple été montré que l'effet dissuasif des caméras ne dépasse pas les deux mois (Mazerolle et al., 2002).

L'analyse des résultats, quant à elle, doit être précise. Pour évaluer les effets dissuasifs, il faut prendre en compte le fait que la vidéosurveillance génère parfois une augmentation des faits portés à la connaissance de la police, ce qui peut fausser l'appréciation sur l'évolution de la délinquance réelle. Par ailleurs, la délinquance étant multiforme, seule l'analyse déployée à l'échelle de différentes catégories de crimes et délits peut être significative des effets réels de la vidéosurveillance : une baisse globale de la délinquance peut masquer une hausse de certains types de faits. De façon similaire, il faut tenir compte à la fois des possibles effets de déplacement de la délinquance et des effets de diffusion des bénéfices de l'installation d'une vidéosurveillance. Une telle prudence est d'autant plus nécessaire que l'équipement en caméras est souvent une mesure parmi d'autres dans un programme plus vaste d'intervention : de ce fait, il s'avère difficile d'isoler l'effet de cette seule action sur la délinquance.

\section{À la recherche de facteurs explicatifs d'une efficacité différentielle}

Les chercheurs ont pu se trouver face à la nécessité de rendre compte de l'échec de certains dispositifs et d'en identifier les facteurs explicatifs. C'est ce qui les a poussés à s'intéresser au processus de mise en œuvre de la vidéosurveillance. Dans le cadre ce que l'on peut appeler le tournant « réaliste » des études évaluatives, amorcé dans les années 1990 par les criminologues britanniques (Tilley, 1993 ; Pawson, Tilley, 1997), les évaluations de la vidéosurveillance sont dès lors passées d'un questionnement sur l'efficacité globale des systèmes de vidéosurveillance à une interrogation sur les conditions de leur fonctionnement. Ces travaux prennent en compte une grande diversité de paramètres touchant au dispositif et à ses cibles mais aussi à la façon dont il est intégré dans une politique de sécurité et utilisé par des opérateurs qui le manipulent au quotidien7. L'analyse du contexte de mise en ouvre de ces dispositifs 
de vidéosurveillance met ainsi en évidence que l'efficacité de ces dispositifs varie selon le type de délit, les caractéristiques du lieu surveillé, les performances techniques du matériel utilisé, les moyens humains en charge de la gestion des équipements (voir les synthèses de Heilmann, Mornet, 2001 ; Heilmann, 2003 ; Le Goff, 2008) ${ }^{8}$.

Concomitamment,le rôle des acteurs dans le processus d'implémentation a été introduit comme une dimension indispensable pour saisir la façon dont fonctionnent les systèmes de vidéosurveillance (Fussey, 2004). Une enquête menée à Londres sur les opérateurs de vidéosurveillance (Norris, Mc Cahill, 2006) montre par exemple que le fonctionnement d'un dispositif dépend non seulement des contextes organisationnels mais aussi des rapports que les acteurs entretiennent au sein d'une même organisation et du caractère plus ou moins conflictuel des relations professionnelles. Peter Manning (2008) soutient, pour sa part, que la culture policière est la matrice à partir de laquelle les technologies vont effectivement être utilisées 9 . À travers la prise en compte des conditions spécifiques de mise en œuvre de la vidéosurveillance dans un contexte d'action particulier, organisé par des règles explicites ou implicites, des rapports de force et des valeurs, ces différents travaux relativisent ainsi la thèse du déterminisme technologique imposé à l'action.

Les workplace studies (Luff, Hindmarsh \& Heath, 2000) et les travaux sur les technologies en action (Heath, Luff, 2000) ${ }^{10}$ s'inscrivent pleinement dans cette logique en réinsérant la vidéosurveillance dans la multiplicité des outils qui peuplent les univers de travail et contribuent à l'accomplissement des activités pratiques. Dans une étude sur le fonctionnement du métro londonien, Heath et Luff (Heath, Luff, 1999 cité par Neyland, 2006, 26-27) ont montré que les opérateurs de vidéosurveillance produisent un travail complexe de construction de la cohérence lorsqu'ils assemblent des fragments de scène, obtenus via des écrans, en interprètent le sens mais font aussi des inférences sur ce qui s'est passé en amont ou ce qui va se passer en aval. Ces travaux relativisent les effets de surveillance en développant l'idée qu'il y a une forme d'incertitude quant à l'existence et l'intelligibilité même de ce qui est observé (voir également Neyland, 2006 ; Smith, 2004 \& 2007). De façon analogue, l'observation du centre de visionnage centralisé des gares néerlandaises conduit à la conclusion que l'agencement technique des dispositifs en limite la capacité de surveillance (Dubbeld, 2005) ${ }^{11}$. D'autres travaux mettent plus largement en évidence des effets de l'utilisation de la vidéosurveillance sur les modes de travail. L'analyse de cas de courses poursuites menées par des policiers sur le terrain mais connectés en permanence à la salle de contrôle de vidéosurveillance montre que leurs perceptions habituelles de la géographie sont bousculées par le fait de disposer d'une série de points de vue distribués dans l'espace mais centralisés au sein de la salle de contrôle. Aux représentations habituelles, basées sur les notions de temps, d'espace et de vitesse, tendent à se substituer de nouvelles représentations de l'espace fondées sur les réseaux de surveillance existants et sur l'interconnexion entre plusieurs points du territoire (Neyland, Kroener, à paraître). Dans une mouvance assez proche, l'approche d'ethnographie institutionnelle qu'adopte Kevin Walby (2005) lui permet de montrer que les opérateurs qu'il étudie (les " travailleurs du contrôle ») ont pleinement adopté les normes de l'organisation qui les emploie. Ce qui ressort de ces différents travaux, est que la vidéosurveillance est partie prenante d'un environnement technologique complexe avec lequel les acteurs sociaux sont en constante interaction et qu'elle participe pleinement à l'élaboration des activités situées de ses utilisateurs directs ou indirects. Il reste cependant difficile de passer du constat d'une telle « intégration » de la vidéosurveillance dans les pratiques à celle d'une efficacité des dispositifs dans la lutte contre la délinquance.

Les nombreux travaux qui se sont intéressés à l'efficacité et, au-delà, aux effets de la vidéosurveillance donnent ainsi une vision relativement claire des effets produits par le recours à la vidéosurveillance sur un territoire donné : sa capacité à prévenir la 
délinquance est limitée. Pourtant l'histoire de la vidéosurveillance ressemble à une success story : le recours à cette technique s'étend et se généralise. Ce paradoxe pourrait s'expliquer par le fait qu'au delà du souci de lutte contre la délinquance, l'installation de caméras de vidéosurveillance s'inscrit dans une logique sociétale plus large. Telle est la thèse d'un certain nombre de travaux critiques, qui dénoncent la vidéosurveillance comme emblématique de la mise en place d'une société de surveillance.

\section{La vidéosurveillance comme technologie de surveillance : la technique, le pouvoir, l'individu}

La majeure partie des études sur la vidéosurveillance relève des surveillance studies, un courant de recherche très présent dans le champ académique du policing $^{12}$. Pour leurs auteurs, la vidéosurveillance, souvent associée à d'autres technologies comme les banques de données ou les dispositifs de traçage, est vue comme une expression technologiquement avancée de la société de surveillance décrite par Foucault (1). D'après ces analyses, le souci d'efficacité dans la lutte contre la délinquance compterait finalement moins que le projet de mise au pas des populations dans le développement de la vidéosurveillance. Si ces travaux mettent bien en évidence les dangers, avérés et potentiels, du recours à la vidéosurveillance, leur capacité à en expliquer la diffusion reste finalement assez limitée (2).

\section{Une surveillance généralisée et centralisée ?}

C'est le sociologue Gary Marx (1985) qui, le premier, a proposé le terme de « nouvelle surveillance » pour caractériser des sociétés dans lesquelles la technologie, en permettant une surveillance totale, a reconfiguré les possibilités mêmes du contrôle social. Ses travaux font directement référence à l'analyse foucaldienne, dans laquelle l'âge des disciplines, qui trouve son apogée au XIXe siècle, repose précisément sur des technologies de surveillance dont le panoptique de Bentham est la figuration la plus emblématique. De nombreux auteurs s'inscrivent dans cette veine et mobilisent au premier degré la référence au dispositif architectural conçu par Bentham pour que les détenus soient surveillés en permanence sans jamais voir ceux qui les observent : la vidéosurveillance est décrite comme une vision moderne du panoptique (Vitalis, 1998), un « panoptique électronique » où l'on est vu sans pouvoir observer en retour (Norris et al., 1998 ; Lyon, 2001). Le parallèle est également souvent fait avec les utopies négatives (dystopia) de certains univers de science fiction, en particulier celui du Big Brother de George Orwell. La « société de surveillance » décrit dès lors une situation dans laquelle des données précises relatives à notre vie privée sont collectées, stockées, récupérées et analysées par d'énormes bases de données informatiques appartenant à de grandes entreprises et services de l'État (Lyon, 1994, 3).

D'autres travaux engagent des discussions sur la pertinence de la figure du panoptique pour décrire la situation actuelle. Métaphore imparfaite, celle-ci continue de proliférer, en particulier à travers la création de formules qui empruntent le mot mais en respécifient la portée par l'ajout d'un qualificatif. On recense ainsi pas moins d'une quinzaine d'expressions dans lesquelles le terme de panoptique est apparié avec divers qualificatifs (Haggerty, 2006). Le panoptique peut par exemple être qualifié de " myope » (myopic panopticon : Leman-Langlois, 2002), au sens où la vidéosurveillance réduirait l'activité criminelle à un comportement directement 
observable et transformerait l'activité policière en une activité strictement réactive face à des faits observés. La référence au panoptique cède aussi parfois la place à la mobilisation d'un autre terme : le « synoptique » (Mathiesen, 1997). Le terme vise à souligner que les technologies modernes permettent à un grand nombre d'individus de diriger leur attention vers quelque chose de commun : la surveillance généralisée s'effectue donc aussi dans le sens d'une surveillance des leaders par l'opinion publique (en particulier via les médias). Dans la même logique, David Lyon (2005), un des auteurs phares des surveillance studies,reconnaît les limites de la théorie selon laquelle les sociétés contemporaines verraient se développer une surveillance généralisée au service du pouvoir : d'une part, il n'y a pas un observateur unique mais une pluralité de lieux d'observation au sein de la société13 ; d'autre part, peu d'individus se sentent oppressés par cette surveillance et même une grande partie d'entre eux collaborent activement à donner des informations sur eux-mêmes, considérant que les bénéfices sont supérieurs aux coûts. Ce constat le conduit alors à mobiliser la notion lacanienne de « scopophilie » (plaisir de regarder) pour décrire la coexistence des dimensions panoptique et synoptique des sociétés contemporaines qu’il qualifie dès lors, à la suite de Mathiesen, de viewer societies (Lyon, 2006).

La critique adressée aux surveillance studies est qu'elles restent souvent au stade d'une réflexion a priori, déconnectée de validations empiriques. Des auteurs comme Gary Marx (2007) ou Peter Manning (2008) reprochent à nombre de travaux leur caractère excessivement globalisant ainsi que leur absence d'ancrage empirique valide. De fait, l'empirie très limitée sur laquelle se fondent ces analyses conduit le plus souvent leurs auteurs à sur-rationaliser le pouvoir et son exercice : les surveillance studies tendent à surévaluer l'efficacité des régimes d'autoritét ${ }^{4}$. Elles attribuent aux outils une finalité politique sans étudier précisément leur genèse et leurs modalités de développement. Ces analyses tendent ainsi à isoler le technique du social, survalorisant les déterminismes technologiques imprimés à l'action. Ces critiques ont en partie porté leurs fruits et, par exemple, sans se distinguer radicalement des surveillance studies, des travaux canadien $\mathrm{s}^{15}$ ou français (Froment, 2009) proposent une vision plus nuancée et plus empiriquement nourrie des effets que produisent les technologies dans les champs de la sécurité et de la justice. D’autres auteurs se refusent à considérer la vidéosurveillance comme une forme dystopique de surveillance maximale en soulignant que la vidéo peut être intrusive et protéger en même temps. C'est la position que défendent Tim Newburn et Stephanie Hayman (2002) à partir de leur étude de la vidéosurveillance installée dans la zone de garde à vue d'un poste de police de Londres. Les deux chercheurs montrent qu'avec ce dispositif, les surveillants sont aussi les surveillés, puisque le dispositif permet un contrôle des actions des représentants de l'État. Les systèmes de surveillance se présentent en fait comme des " assemblages de surveillance » (Ericson, Haggerty, 2000)dans lesquels des technologies, des pratiques et des acteurs multiples s'associent pour produire du contrôle dans les espaces publics. Si la surveillance se généralise, elle n'est pas complètement centralisée, dans la mesure où les réticences institutionnelles au partage de l'information et les spécificités des logiciels utilisés empêchent l'intégration des systèmes de surveillance (Ericson, Haggerty, 2006 ; Manning, 2008). Enfin, les analyses ethnométhodologiques éventuellement combinées avec d'autres approches (Neyland, 2006, 38-45) relativisent la force de cette surveillance dans la mesure où elles montrent que le contenu des images diffusées en temps réel, le sens même qu'il faut donner à ce qui se passe sur l'écran n'est ni explicite, ni univoque : il est produit au fil de négociations locales et situées auxquelles participent plusieurs types d'acteurs comme les opérateurs et les forces de police avec lesquels ils sont en contact (Neyland, 2006, 19-45). La métaphore du panoptique est ainsi remise en cause parce qu'elle laisse de côté des pans entiers du processus de surveillance tel qu'il s'incarne dans les pratiques (Haggerty, 2006). 


\section{Une mise en évidence des dangers plus qu'une analyse des logiques de développement}

Les surveillance studies proposent une approche critique des nouvelles techniques disponibles - dont la vidéosurveillance fait partie - en soulignant les effets pervers et les dangers d'une surveillance déployée à grande échelle dans la mesure où elle permet le contrôle des individus à distance (Lyon, 2005). La principale préoccupation de ce courant concerne donc les libertés individuelles, possiblement mises à mal par l'intensification de la surveillance : l'expansion de la vidéosurveillance est vue comme une étape supplémentaire franchie dans la remise en cause des libertés, en particulier à la suite des attentats du 11 septembre 2001 (Ericson, Haggerty, 2006).

Certains travaux relevant des surveillance studies tentent toutefois d'aller au delà de la dénonciation générale d'une atteinte à la liberté d'aller et venir et mettent en avant des dangers liés à l'usage de la vidéosurveillance pour dénoncer le modèle social sous-jacent au recours à la vidéosurveillance. Des études ont ainsi mis en évidence l'existence de pratiques discriminatoires et en tirent la conclusion que la vidéosurveillance serait délibérément employée pour surveiller certaines catégories de population. C'est ce que montrent Clive Norris et Gary Armstrong (1999a) suite à l'observation de trois centres de supervision de la vidéosurveillance en Angleterre. Ils ont examiné comment les opérateurs développent, avec la pratique, un ensemble de règles opérationnelles pour réduire la population générale à celle des suspects, ce qui les conduit de fait à exercer des pratiques de surveillance discriminatoires. Par exemple, le port d'une capuche ou d'une casquette est systématiquement interprété par les opérateurs comme la preuve d'une volonté d'échapper au regard des caméras, donc comme démontrant une intention criminelle. Les deux chercheurs calculent en outre que « les Noirs » ont entre 1,5 et 2,5 fois plus de chances d'être ciblés que ne le laisserait supposer leur pourcentage dans la population, contrairement aux femmes, une population vulnérable et pourtant largement délaissée par le regard de la caméra. En ciblant des catégories particulières de population, la vidéosurveillance renforce la coupure entre « inclus » et « exclus »; en ce sens elle est au service d'un certain ordre social. Telle est la thèse à laquelle aboutissent un grand nombre de travaux. Ainsi, Roy Coleman et Joe Sim, qui ont étudié la mise en œuvre de la vidéosurveillance dans le centre de Liverpool, considèrent la vidéosurveillance comme l'un des aspects coercitifs du pouvoir, dirigés vers les insoumis à l'ordre néolibéral, autrement dit comme un outil utilisé pour imposer un nouvel ordre social (Coleman, Sim, 2000, 634). Au Pays de Galles, où ils ont étudié les dispositifs de deux petites villes (Aberystwyth et Cardigan), Katherine Williams et Craig Johnstone(2000)concluent à un malling (fractionnement) de l'espace public, et non à un panoptique au sens de Bentham, dans la mesure où les caméras sont le vecteur d'un « regard sélectif ». La vidéosurveillance produit donc des effets d'exclusion aussi bien dans les centres commerciaux (shopping malls) que dans l'espace public, avec toutefois d'importantes variations suivant les sites comme le montre une étude réalisée à Oslo (Lomell, 2004).

C'est donc plus en termes d'effets produits qu'en termes de logiques et de processus de développement que les surveillance studies apportent des éléments de validation empirique. Ce sont d'autres travaux, moins focalisés sur la dénonciation des risques de la vidéosurveillance, qui portent leur attention sur les processus de genèse et de diffusion des dispositifs de vidéosurveillance.

\section{La vidéosurveillance comme outil d'action publique : processus de}




\section{genèse et de diffusion}

C'est l'ancrage dans une perspective de sociologie de l'action publique, au sens large, qui apporte finalement le plus de réponses à la question du développement de la vidéosurveillance de voie publique. Ces travaux, qui traitent de manière disparate de situations nationales et locales contrastées, permettent malgré tout de mettre en évidence les conditions de production des dispositifs (1) mais aussi de décortiquer les processus par lesquels la vidéosurveillance se généralise et se banalise (2).

\section{Les conditions de production des dispositifs de vidéosurveillance de voie publique}

Les villes britanniques sont l'objet de nombreux travaux, du fait de l'important déploiement de caméras de vidéosurveillance en Grande-Bretagne. Ces travaux auxquels font écho des études menées dans d'autres pays et parfois des tentatives de synthèse à l'échelle régionale (par exemple européenne : Norris, Mc Cahill, Wood, 2004) - ont mis en évidence un certain nombre de facteurs sociaux, politiques et juridiques qui favorisent le développement de la vidéosurveillance de voie publique.

Le développement de la vidéosurveillance est bien souvent rapporté uniquement au contexte social général : hausse de la délinquance, baisse de la confiance dans le système judiciaire, émergence d'une société du risque telle que théorisée par Ulrich Beck, développement d'une conception actuarielle de la justice... Des facteurs politiques et institutionnels sont également régulièrement avancés. Ainsi, tandis que N. Fyfe et J. Bannister (1996) associent le développement de la vidéosurveillance à la New Right Law and Order Policy, P. Fussey (2008) voit dans l'avènement de la stratégie de réduction du crime du New Labour le principal facteur de développement de la vidéosurveillance en Grande-Bretagne. Au delà de la couleur politique du gouvernement, c'est l'évolution des modes de gouvernement qui favorise le développement de la vidéosurveillance. D’un côté, la mise en œuvre des principes du new public management dans les services de l'État conduit à la définition de priorités quantifiables et au développement d'indicateurs de performance, ce qui engage les services de police dans une voie rationaliste consistant à générer des réponses stratégiques aux problèmes identifiés par la recherche et l'évaluation. D’un autre côté, les partenariats locaux associant acteurs publics et privés sont encouragés pour lutter contre des problèmes définis localement (sentiment d'insécurité, petits désordres et délits répétés) (Mackay, 2003 ; Fussey, 2008) ; la participation de professionnels porteurs de savoirs criminologiques orthodoxes aux partenariats locaux prédispose alors ces derniers à considérer la vidéosurveillance comme le meilleur moyen d'action parmi l'éventail de mesures disponibles ${ }^{16}$, ce d'autant plus que le gouvernement britannique finance les installations de vidéosurveillance ${ }^{17}$. Le rôle de l'État central n'apparaît pas toujours aussi déterminant : dans d'autres pays, les initiatives viennent principalement des gouvernements locaux. C'est le cas en Australie, où ils sont néanmoins soutenus - financièrement et méthodologiquement - par les gouvernements des États fédérés (Wilson, Sutton, 2004), mais également en France. Poussés à la fois par l'État, qui propose une contractualisation en la matière, et par des administrés qui interpellent des municipalités renforcées par la décentralisation (Le Goff, 2005 ; Germain 2008), les maires français se sont emparés des questions de sécurité et ont cherché à développer des outils qui leur soient propres, parmi lesquels la vidéosurveillance. Ce n'est qu'à partir de 2007 que l'État français a lancé un programme de soutien à l'équipement des villes.

Les règles juridiques font également partie des éléments pris en compte dans les analyses de la diffusion de la vidéosurveillance. En Grande-Bretagne, l'expansion de 
la vidéosurveillance est facilitée par une absence d'encadrement juridique ${ }^{18}$. La loi relative à la justice criminelle et à l'ordre public (1994) donne aux autorités locales en Angleterre et au Pays de Galles le pouvoir de se doter d'équipements de vidéosurveillance sans autorisation préalable ; ce sont les autorités qui se chargent elles-mêmes d'élaborer des codes de bonne conduite ${ }^{19}$. En France, les analyses juridiques de la vidéosurveillance, qu'il s'agisse d'études commanditées par le pouvoir politique (Sérusclat, 1995) ou la $\mathrm{CNIL}^{20}$ (Cadoux, 1993) ou de commentaires universitaires ${ }^{21}$, montrent que le régime d'autorisation administrative préalable mis en place en 1995 et modifié ensuite (Froment, 2006) apparaît plus comme un moyen de légitimation du recours à la vidéosurveillance que comme une véritable contrainte.

Un autre facteur susceptible de favoriser la diffusion de la vidéosurveillance est le soutien de la population, d'ailleurs revendiqué par les promoteurs des projets de vidéosurveillance (Ditton, 2000). Les travaux qui se sont intéressés de près aux opinions relatives à la vidéosurveillance soulignent toutefois qu'il s'agit d'opinions peu informées et relativement ambiguës ${ }^{22}$. Ainsi, une étude menée à Cardiff, Bristol, Birmingham et Coventry (Charman, Honess, 1992) révèle un fort soutien de la population à la vidéosurveillance ( $85 \%$ d'avis favorables) mais précise qu'il repose sur une méconnaissance des capacités réelles des dispositifs. Le soutien de la population à la vidéosurveillance apparaît en outre assez paradoxal : d'après l'enquête européenne "Urbaneye ", si les enquêtés soutiennent majoritairement la vidéosurveillance, ils sont aussi plus de la moitié à lui prêter une efficacité limitée (Hempel, Töpfer, 2004). Une étude canadienne qui a utilisé la technique des focus groups pour saisir les perceptions et représentations de la vidéosurveillance (LemanLanglois, 2008) rapporte des conclusions similaires : les enquêtés réclament davantage de caméras tout en indiquant que les caméras actuellement en place ne changent pas vraiment le niveau de délinquance de leur quartier. Enfin, une part des opinions sur la vidéosurveillance apparaît réversible, comme le montre une étude menée auprès des résidents de huit zones équipées en vidéosurveillance (Gill et al., 2007). L'enquête d'opinion révèle une diminution du soutien public une fois le dispositif expérimenté. Le soutien de la population n'est donc pas un facteur univoque et doit être analysé en lien avec les stratégies de légitimation du recours à la vidéosurveillance.

Cette dernière remarque met au jour la nécessité d'aller au-delà de l'énumération des différents facteurs favorisant le recours à la vidéosurveillance ${ }^{23}$, énumération qui laisse dans l'ombre les processus qui conduisent in fine à cette décision. Autrement dit, reste à comprendre comment ces différents éléments interagissent.

\section{De la recherche de causes à l'analyse de processus}

Certaines recherches sur la vidéosurveillance se sont montrées attentives aux processus par lesquels, dans un contexte donné, le recours à la vidéosurveillance était envisagé, concrétisé et consolidé. Au moins deux schémas d'analyse peuvent être isolés au sein de ces travaux : celui des coalitions d'acteurs et celui de la logique de l'innovation, inspiré des études de sociologie des sciences et techniques.

Ce sont surtout les travaux britanniques qui mettent en avant l'importance des coalitions d'acteurs associés dans l'avènement d'un projet de vidéosurveillance : autorités locales, polices publiques, commerces, services privés de sécurité. Des études portant sur Glasgow (Fyfe, Bannister, 1996 ; Mackay, 2003) ou Liverpool (Coleman, Sim, 1998, 2000) soulignent ainsi que c'est une alliance stratégique entre gouvernement local et intérêts économiques locaux qui est à l'origine de l'installation de la vidéosurveillance de voie publique. Ces deux catégories d'acteurs cherchent à rendre leur ville plus attractive en réduisant la délinquance pour mieux asseoir son 
développement économique, tandis que la police, percevant la vidéosurveillance comme un outil de management opérationnel à moindre coût, apporte son soutien à l'initiative (Williams, Johnstone, 2000 ; Mackay, 2003). Ce type de schéma a aussi été appliqué au cas français : s'appuyant sur les analyses de B. Rochette et E. Marchandet (1998), Frédéric Ocqueteau (2004) parle de légitimation croisée entre opérateurs de réseaux et collectivités territoriales, la coalition associant ici fournisseurs de technologie et autorités locales.

Dans une autre perspective, des études se sont intéressées à la vidéosurveillance en essayant de tirer parti des acquis de la sociologie des sciences et des techniques, de ses apports théoriques mais aussi des résultats produits à partir de l'analyse d'autres types de technologies que celles de surveillance. Une première série de travaux insiste sur l'invention des usages : au delà des intentionnalités initiales, c'est la façon dont l'outil est approprié qui le fait vivre. En ce sens, les technologies de sécurité n'échappent pas à l'ample travail de négociation dans lequel s'engagent les différents acteurs qui sont associés au dispositif et qui le font exister (Akrich, Méadel, 1996, 56). Dans cette logique, la recherche de Frédéric Ocqueteau et Marie-Lys Pottier sur un centre commercial (Ocqueteau, Pottier, 1995) a mis en évidence le déploiement d'usages « imprévus » de la vidéosurveillance. Le dispositif est par exemple intégré dans une tactique de gestion des incidents avec de possibles mauvais payeurs ${ }^{24}$ alors qu'il était initialement conçu pour repérer la préparation d'opérations délictuelles. Concernant la vidéosurveillance de voie publique, tandis que la fonction affichée est le plus souvent la dissuasion en vue de réduire le taux de criminalité, les usages effectifs sont marqués par le souci de repérage et d'identification des criminels (Douillet, Dumoulin, Germain, 2011, à paraître). Clive Norris et Gary Armstrong (1999b) proposent le concept d' " expandable mutability » pour souligner le fait que des caméras de surveillance installées dans un objectif particulier sont en fait utilisées pour d'autres usages.

Les modes de raisonnement de la sociologie des sciences, et en particulier de la sociologie de la traduction, permettent aussi de mettre en évidence les processus d'enrôlement qui alimentent le développement de la vidéosurveillance. À partir d'une étude relative aux usages de la vidéosurveillance dans trois villes françaises (Rochéet al., 2007), nous nous sommes ainsi intéressés aux mécanismes d'adhésion à la vidéosurveillance, montrant que l'usage de la technologie est susceptible d'enrôler un certain nombre d'acteurs : ses utilisateurs, d'une part, via des mécanismes d'appropriation, mais également ses détracteurs, par un affaiblissement des oppositions (Douillet, Dumoulin, Germain, 2011, à paraître). Trois mécanismes d'appropriation de la vidéosurveillance par les acteurs en charge de son utilisation ont ainsi été mis en évidence. Tout d'abord, la spécialisation de services en charge de la gestion de la vidéosurveillance et/ou du visionnage des images fait de ces nouveaux professionnels des alliés du développement de la vidéosurveillance. Ensuite, les gratifications symboliques qui peuvent être retirées de l'usage de la vidéosurveillance contribuent à l'enrôlement d'acteurs comme les policiers municipaux, ravis d'intégrer à leur activité une dimension « investigation » qui les rapproche de la police judiciaire (nationale). Enfin, la police nationale apprécie la " ressource à moindre coût » que constitue la vidéosurveillance puisqu'elle peut avoir la main sur un dispositif qu'elle n'a en rien financé et le mobiliser selon ses propres priorités professionnelles : maintien de l'ordre ou investigation judiciaire notamment. Parallèlement aux processus d'appropriation de l'outil par ses utilisateurs, le développement même du dispositif affaiblit les oppositions et la découverte d'usages chemin faisant démultiplie les arguments de légitimation, nourrissant ainsi le processus d'enrôlement. L'extension du système de vidéosurveillance réduit par exemple la portée de l'argument sur les effets de déplacement tandis que la mise en place d'un comité d'éthique auquel les opposants acceptent de participer les inclut de fait dans le dispositif ${ }^{25}$. Les usages développés au fil de la pratique - identification 
d'auteurs de crimes et délits, calibrage des réponses policières et gestion des petits désordres dans une perspective pleine et entière de police administrative (Froment, 2006, 439) - permettent en outre de présenter la vidéosurveillance non seulement comme un instrument de dissuasion, mais aussi comme un soutien logistique et un instrument de répression. La vidéosurveillance est finalement légitimée par sa multifonctionnalité.

\section{Conclusion}

La diffusion de la vidéosurveillance apparaît largement déconnectée de sa capacité à lutter contre la délinquance tandis que son inscription dans un projet global de surveillance des populations reste difficile à prouver. Le processus de diffusion de la vidéosurveillance résulte plutôt de la conjonction de plusieurs facteurs : souci des principales forces politiques d'afficher une préoccupation sécuritaire, appropriation de la thématique par les autorités locales, volonté politique nationale de renforcer les partenariats psublic-privé au niveau local (Grande-Bretagne), développement d'un marché de la sécurité, intérêts commerciaux... La vidéosurveillance peut être analysée comme un dispositif sociotechnique capable d'agréger différents types d'acteurs, porteurs d'intérêts spécifiques divers. En tant qu'acteur-réseau, la vidéosurveillance se révèle aussi capable d'enrôler des acteurs par différents mécanismes, au delà du cercle de ses promoteurs initiaux. Ses utilisateurs s'approprient l'objet et découvrent chemin faisant de multiples usages, qui deviennent alors autant de justifications à son emploi, alimentant par là même le processus d'enrôlement sur un site.

Une question demeure toutefois en suspens après cette revue de la littérature internationale sur la vidéosurveillance : la diffusion de ces dispositifs traduit-elle l'avènement d'une nouvelle pénologie ? Ce questionnement, soulevé par les surveillance studies en particulier, est loin d'être dénué d'intérêt à l'heure où les villes, marquées par une hétérogénéité croissante, constituent un terreau propice à la peur de l'autre (Bannister et al., 1998). Seules des observations ethnographiques systématiques dans des centres de supervision urbaine permettraient de répondre à cette question. Si des études de ce type ont été menées dans différents pays (voir Norris, Armstrong, 1999a, Norris, Wilson, 2006 et Neyland, 2006 pour la GrandeBretagne ; Dubbeld, 2003 pour les Pays-Bas ; Walby, 2005 pour le Canada), il n'en existe pas pour l'heure en France. La confrontation des usages de la vidéosurveillance effectivement observés et des suites judiciaires données aux cas repérés permettrait d'examiner l'hypothèse d'une extension des capacités de surveillance de l'État et celle de l'avènement d'une nouvelle forme de contrôle social (façonnée par les municipalités) focalisée sur la gestion de catégories de population et de comportements particuliers. Une telle approche permettrait aussi de tirer des conclusions affermies et inédites sur un point de la littérature resté aveugle : la capacité effective de normalisation des conduites de la vidéosurveillance.

\section{Bibliographie}

Akrich M., Méadel C., 1996, Anthropologie de la télésurveillance en milieu privé, Rapport pour le Pirvilles-CNRS et l'IHESI, Paris, Centre de sociologie de l'innovation.

Bannister I., Fyfe R., Kearn A., 1998, CCTV and the city, in Norris C., Moran J., Armstrong G. (Eds), Surveillance, Closed Circuit Television and Social Control, Aldershot, Ashgate, 2139.

Bauer A., Freynet F., 2008, Vidéosurveillance et vidéoprotection, Paris, PUF (coll. "Que sais-je ?").

Boullier D., 1995, La vidéosurveillance à la RATP : un maillon controversé de la chaîne de production de sécurité, Les Cahiers de la sécurité intérieure, 21, 88-100. 
Brown B., 1995, CCTV in Town Centres: Three Case Studies, Crime Prevention Unit Series, Paper $\mathrm{n}^{\circ}$ 68, Londres, Home Office.

Buclos M., Sarno C., 1996, Codes of practice and public closed circuit television systems, Londres, Local Government Information Unit.

Cadoux L., 1993, Vidéosurveillance et protection de la vie privée et de libertés fondamentales, Rapport du 30 novembre 1993, Paris, CNIL.

Charman T., Honess E., 1992, Closed circuit television in public spaces: its acceptability and perceived effectiveness, Crime Prevention Unit Series, Paper $n^{\circ} 35$, Londres, Home Office Police Department, Police Research Group.

Chatterton M. R., Frenz S. J., 1994, Closed-circuit television: its role in reducing burglaries and the fear of crime in sheltered accommodation for the elderly, Security Journal, 5 (3), 133-139.

Coleman R., Sim J., 1998, From the dockyards to the Disney store: surveillance, risk and security in Liverpool city centre, International Review of Law Computers and Technology, $12(1), 27-45$.

Coleman R., Sim J., 2000, 'You'll never walk alone' : CCTV surveillance, order and neoliberal rule in Liverpool city, British Journal of Sociology, 51 (4), 623-639.

Cusson M., 2005, La surveillance et la télésurveillance : sont-elles efficaces ?, Revue Internationale de Criminologie et de Police Technique et Scientifique, LVIII, 131-150.

Darras E., Deharbe D., 1996, La politique du regard. Remarques sur la légalisation de la vidéosurveillance, in CURAPP, La gouvernabilité, Paris, PUF, 77-90.

Devresse M.-S., Pieret J. (dir.), 2009, La vidéosurveillance, entre usages politiques et pratiques policières, Bruxelles, CEP-Politeia.

Ditton J., 1998, Public Support for Town Centre CCTV. Schemes: Myth or Reality?, in Norris C., Moran J., Armstrong G. (Eds), Surveillance, Closed Circuit Television and Social Control, Aldershot, Ashgate, 221-228.

Ditton J., 2000, Crime and the City: Public attitudes towards open-street CCTV in Glasgow, British Journal of Criminology, 40 (4), 692-709.

Ditton J., Short E., 1998, Seen and now heard. Talking to the targets of open-street CCTV, British Journal of Criminology, 37, 3, 404-428.

Douillet A.-C., Dumoulin L., Germain S., (printemps 2011, à paraître), Un dispositif sociotechnique à la loupe : le développement de la vidéosurveillance dans trois villes françaises, Quaderni.

Dubbeld, L., 2003, Observing bodies. Camera surveillance and the significance of the body, Ethics and Information Technology, 5, 151-162.

Dubbeld L., 2005, The Role of Technology in Shaping CCTV Surveillance Practices, Information, Communication \& Society, 8 (1), 84-100.

Ericson R. V., Haggerty K. D., 2000, The Surveillant Assemblage, The British Journal of Sociology, 51 (4), 605-620.

Ericson R. V., Haggerty K. D., 2006, The new politics of surveillance and visibility, inEricson R. V., Haggerty K. D. (Eds), The New Politics of Surveillance and Visibility, Toronto, University of Toronto Press, 3-25.

Favoreu L., 1995, Décision du 18 janvier sur la loi d'orientation et de programmation relative à la sécurité, Revue française de droit constitutionnel, 22, 362-372.

Forest D., 1999, Vidéosurveillance dans les lieux publics : législation effective, application transparente ?, Quaderni, 40, 31-51.

Froment J.-C., 2006, Regard juridique sur la vidéosurveillance urbaine : un droit en trompe-l'œil, La semaine juridique, Édition Adm. et Collec. Terr., 13, 435-440.

Froment J.-C., 2009, Exécution des peines, techniques et technologies. Évolution des fondements de la pénalité et rationalités politiques, in Collectif, L'exécution des décisions en matière pénale en Europe. Du visible à l'invisible, Actes du colloque international tenu à Lyon en décembre 2008, Ministère de la Justice, 142 et suiv.

Fussey, P., 2004, New Labour and New Surveillance: Theoretical and Political Ramifications of CCTV Implementation in the UK, Surveillance \& Society, 2 (2/3), 251-269.

Fussey P., 2008, Beyond liberty, beyond security: the politics of public surveillance, British Politics, 3 (1), 120-135.

Fyfe N. R., Bannister J., 1996, City watching: closed circuit television surveillance in public spaces, Area, 28 (1), 37-46.

Germain S., 2008, Les politiques locales de sécurité en France et en Italie. Une 
comparaison des villes de Lyon, Grenoble, Bologne et Modène, Thèse pour le doctorat de science politique, Grenoble, Université Pierre Mendès-France.

Gill M., Spriggs A., 2005, Assessing the Impact of CCTV, Home Office Research Study n ${ }^{\circ}$ 292, Londres, Home Office Research, Development and Statistics Directorate.

Gill M., Turbin V., 1999, Evaluating 'Realistic Evaluation': evidence from a study of CCTV, Crime Prevention Studies, 10, 179-199.

Gill M. et al., 2007, Public perceptions of CCTV in residential areas. 'It is not as good as we thought it would be', International Criminal Justice Review, 17 (4), 304-324.

Graham, S., 1998, Towards the fifth utility? On the extension and normalisation of public CCTV, in Norris C., Moran J., Armstrong G. (Eds), Surveillance, closed circuit television and social control, Ashgate, Aldershot, 89-112.

Grandmaison R., Tremblay P., 1997, Évaluation des effets de la télésurveillance sur la criminalité commise dans 13 stations du métro de Montréal, Criminologie, 30 (1), 93-110.

Haggerty K. D., 2006, Tear down the walls: on demolishing the panopticon, in Lyon D. (Ed.), Theorizing surveillance. The Panopticon and Beyond, Cullompton, Willan Publishing, 23-45.

Heath C., Luff P., 1999, Surveying the scene: The monitoring practices of staff in control rooms in Noyes J., Barnsby M. (Eds), Proceedings of People pits and command centres, IEE Press, University of Bath (UK), p.1-6.

Heath C., Luff P., 2000, Technology in Action, Cambridge, Cambridge University Press.

Heilmann É., 2003, La vidéosurveillance, une réponse efficace à la criminalité ?, Criminologie, 36 (1), 89-102.

Heilmann, E., Mornet, M.-N., 2001, L'impact de la vidéosurveillance sur les désordres urbains, le cas de la Grande-Bretagne, Les Cahiers de la Sécurité Intérieure, 46 (4), 197211.

Hempel L., Töpfer E., 2004, CCTV in Europe. Final Report, The Urbaneye Working Papers Series, edited by $5^{\text {th }}$ Framework Programme of the European Commission, Berlin, Centre for Technology and Society.

Hier S., Greenberg J., 2007, Surveillance Studies Reader, Buckingam, Open University Press.

Le Goff T., 2005, L’insécurité saisie par les maires, Revue française de science politique, 55 (3), 415-444.

Le Goff T. (dir.), 2008, Vidéosurveillance et espaces publics. État des lieux des évaluations menées en France et à l'étranger, Paris, Institut d'aménagement et d'urbanisme d'Île-deFrance.

Leman-Langlois S., 2002, The myopic Panopticon: The Social Consequences of Policing through the Lens, Policing and Society, 13 (1), 44-58.

Leman-Langlois S., 2008, The local impact of police videosurveillance, in Leman-Langlois S. (Ed.), Technocrime, Willan Publishing, Portland, 27-45.

Lianos M., 1996, La poétique de la peur. Le sujet hyper-régulier, thèse pour le doctorat de sociologie, Paris, Université Paris VII.

Lomell H.M., 2004, Targeting the Unwanted: Video Surveillance and Categorical Exclusion in Oslo, Norway, Surveillance \& Society, 2 (2/3), 346-360.

Luff P., Hindmarsh J., Heath C., 2000, Workplace Studies, Cambridge, Cambridge University Press.

Lyon D., 1994, The Electronic Eye. The rise of the surveillance society, Cambridge, Polity Press.

Lyon D., 2005 [2001], Surveillance Society. Monitoring everyday life, Buckingham, Open University Press.

Lyon D., 2006, 9/11, Synopticon, and Scopophilia : Watching and Being Watched, in Ericson R.V., Haggerty K. D. (Eds), The New Politics of Surveillance and Visibility, Toronto, University of Toronto Press, 35-54.

Lyon D., 2007, Surveillance Studies. An overview, Cambridge, Polity Press.

Mackay D., 2003, Multiple targets: the reasons to support town-centre CCTV systems, in Gill M. (Ed.), CCTV, Leicester, Perpetuity Press, 23-35.

Manning P. K., 2008, A view of surveillance, in Leman-Langlois S. (Ed.), Technocrime, Portland, Willan Publishing, 209-242.

Marx G. T., 1985, 'I'll be Watching You': Reflections on the New Surveillance, Dissent, 
Winter, 26-34.

Marx G. T., 2007, Desperately Seeking Surveillance Studies: Players in Search of a Field, Contemporary Sociology, 36 (2), 125-130.

Mathiesen T., 1997, The Viewer Society: Michel Foucault's 'Panopticon', Theoretical Criminology, 1 (2), 215-234.

Mazerolle L., Hurley D., Chamlin M., 2002, Social behavior in public space: an analysis of behavioral adaptation to CCTV, Security Journal, 15 (3), 59-75.

Newburn T., Hayman S., 2002, Policing, surveillance and social control. CCTV and police monitoring of suspects, Cullompton, Willan Publishing.

Neyland D., 2006, Privacy, Surveillance and Public Trust, Basingstoke, Palgrave Macmillan.

Neyland D., Kroener I., à paraître, Cut to the chase: editing time and space through CCTV, Droit et cultures.

Norris C., Armstrong G., 1999a, CCTV and the social structuring of surveillance, Crime Prevention Studies, 10, 157-178.

Norris C., Armstrong G., 1999b, The Maximum Surveillance Society, New York, Berg.

Norris C., Mc Cahill M., 2006, CCTV: Beyond Penal Modernism?, British Journal of Criminology, 46, 97-118.

Norris C., McCahill M., Wood D., 2004, The growth of CCTV: a global perspective on the international diffusion of videosurveillance in publicly accessible space, Surveillance and society, 2 (2/3), 110-135.

Norris C., Moran J., Armstrong G., 1998, Surveillance, Closed Circuit Television and Social Control, Aldershot, Ashgate.

Norris C., Wilson D., 2006, Surveillance, Crime and Social Control, Aldershot, Ashgate.

Ocqueteau F., 2001, Cinq ans après la loi "vidéosurveillance" en France, que dire de son application?, Urbanisme et sécurité, 43, 101-110.

Ocqueteau F., 2004, Polices entre Etat et marché, Paris, Presses de Sciences Po.

Ocqueteau F., Heilmann E., 1997, Droit et usages des nouvelles technologies : les enjeux d'une réglementation de la vidéosurveillance, Droit et Société, 36-37, 331-344.

Ocqueteau F., Pottier M.-L., 1995, Vigilance et sécurité dans les grandes surfaces, Paris, L'Harmattan.

Pawson R., Tilley, N., 1997, Realistic Evaluation, Londres, Sage.

Pécaud D., 2002, L’impact de la vidéosurveillance sur la sécurité dans les espaces publics et les établissements recevant du public, Paris, Rapport IHESI.

Pellet R., 1995, La vidéosurveillance et l'application de la loi informatique et libertés, Revue administrative, 285, 142-151.

Roché S., Douillet A.-C., Dumoulin L., Germain S., Cameratti, N., 2007, Les usages techniques et politiques de la vidéosurveillance : une comparaison entre Lyon, SaintÉtienne et Grenoble, Rapport final pour l'INHES, Grenoble, UMR Pacte.

Rochette B., Marchandet E., 1998, Vidéosurveillance et télésurveillance, médiations techniques et médiations politiques, in Ansidei M., Dubois D., Fleury D., Munier B. (dir.), Les risques urbains : acteurs, systèmes de prévention, Paris, Anthropos, 185-206.

Sérusclat F., 1995, Les nouvelles techniques d'information et de communication : l'homme cybernétique?, Office parlementaire d'évaluation des choix scientifiques et technologiques, Assemblée Nationale/Sénat.

Smith G., 2004, Behind the Screens: Examining Constructions of Deviance and Informal Practices among CCTV Control Room Operators in the UK, Surveillance \& Society,2 (2/3), 376-395.

Smith G., 2007, Exploring Relations between Watchers and Watched in Control(led) Systems: Strategies and Tactics, Surveillance \& Society, 4 (4), 280-313.

Tilley N., 1993, Understanding car parks, crime and CCTV: Evaluation lessons from safer cities, Crime Prevention Unit Series, Paper $\mathrm{n}^{\circ}$ 42, Londres, Home Office Police Department.

Vitalis A., 1998, De la relation de discipline aux techniques de contrôle, in Ansidei M., Dubois D., Fleury D., Munier B. (dir.), Les risques urbains. Acteurs et systèmes de prévention, Paris, Anthropos, 173-183.

Walby K., 2005, How closed-circuit television surveillance organizes the social: an institutional ethnography, The Canadian Journal of Sociology, 30 (2), 189-214. 
Webb B., Laycock G., 1992, Reducing crime on the London underground. An evaluation of three pilot projets, Crime Prevention Unit Series, Paper $n^{\circ} 30$, Londres, Home Office.

Welsh B., Farrington D., 2008, Effects of Closed Circuit Television Surveillance on Crime, Campbell Systematic Reviews, 17, 2-73.

Williams C. A., 2003, Police surveillance and the emergence of CCTV in the 1960s, in Gill M. (Ed.), CCTV, Leicester, Perpetuity Press, 9-22.

Williams K., Johnstone C., 2000, The politics of the selective gaze: closed circuit television and the policing of public space, Crime, Law and Social Change, 34 (2), 183-210.

Wilson D., Sutton A., 2004, Watched over or over-watched? Open street CCTV in Australia, Australian and New Zealand Journal of Criminology, 37 (2), 211-230.

\section{Notes}

1 Pour un panorama général mais un peu daté, voir Norris et al., 2004.

2 C'est début 2008 que le basculement est opéré : la commission nationale de la vidéosurveillance mise en place fin 2007 débouche sur un « plan vidéoprotection » ( $L e$ Monde, 16 février 2010).Ce basculement sémantique vise à détacher l'installation de caméras sur la voie publique de l'idée négative de surveillance et d'atteinte aux libertés individuelles pour l'associer à celle de sécurité, comme en atteste le slogan choisi : « La sécurité au service de la liberté ». Voir Bauer, Freynet, 2008, dont le titre est précisément Vidéosurveillance et vidéoprotection.

3 Par exemple en France, dans le travail pionnier de Pécaud, 2002.

4 Les travaux considérés comme des références en Grande-Bretagne (Webb, Laycock, 1992 ; Tilley, 1993 ; Gill, Spriggs, 2005) ont tous été financés par le Home Office, qui promeut le développement de la vidéosurveillance.

5 Sur les 92 travaux examinés par B. Welsh et D. Farrington (2008), seuls 44 d'entre eux répondaient aux critères qui garantissent, pour les deux auteurs, une étude méthodologiquement fiable. Et seules 41 études ont finalement fait l'objet de la métaanalyse.

6 Par exemple, comparer un quartier du centre-ville avec le reste de la ville n'est pas forcément pertinent.

7 Sur les difficultés de l'approche réaliste, voir Gill, Turbin, 1999.

8 S'efforçant de synthétiser et d'articuler les conclusions de nombreux travaux, Maurice Cusson (2005) a formulé huit propositions explicatives de l'efficacité différentielle de la vidéosurveillance. 1. Les délinquants déterminés tentent de découvrir les forces et faiblesses des dispositifs et agissent en conséquence. 2. La vidéosurveillance peut faire baisser la plupart des délits mais obtient ses meilleurs résultats sur les délits visibles commis par des délinquants craignant la confrontation. 3. C'est dans les lieux fermés d'où les délinquants ne peuvent fuir facilement une fois leur coup fait que la vidéo est la plus performante. 4. Un dispositif de vidéo qui augmente réellement la capacité du personnel de sécurité à détecter et à intervenir offre d'excellentes chances de faire reculer durablement la délinquance visée. 5. Il arrive que la vidéo produise un effet anticipé et qu'avec le temps son efficacité tende à s'estomper. 6. La publicité amplifie les résultats à court terme de la vidéo. 7 . La vidéo a d'excellentes chances de produire un effet marqué et durable là où le volume des infractions est élevé et quand elle est utilisée pour intervenir régulièrement. 8. La vidéo agit moins par l'arrestation des suspects qu'en leur faisant honte ou en les conduisant à réviser à la hausse leur perception des risques.

9 Voir aussi les réflexions et retours d'expérience sur les usages en contexte policier, belge et français (Devresse et Pieret, dir., 2009).

10 Ces recherches, de type ethnographique, inspirées de l'ethnométholologie, mettent l'accent sur la façon dont les activités impliquant des individus, des technologies et des objets sont coordonnées, performées et rendues intelligibles en situation.

11 Les limites mises en évidence sont les suivantes : distance entre le centre et les forces de police susceptibles d'intervenir ; grand nombre de caméras pour peu d'opérateurs ; variété des tâches assurées par les opérateurs ; soucis techniques fréquents (caméras, téléphones, talkies-walkies) ; détérioration du matériel (toiles d'araignée, friture, vandalisme...). Voir aussi le travail réalisé en France à la RATP (Boullier, 1995).

12 Entre 1960 et 1969, la base Sociological Abstracts ne comptait que 6 articles avec pour mot-clé "surveillance », alors qu'entre 1990 et 1999, il y en 563 (Marx, 2007). Cet ensemble de recherches tend de plus à s'institutionnaliser avec la publication de Readers (Norris, Wilson, 2006 ; Hier, Greenberg, 2007 ; Lyon, 2007) mais aussi avec la création en 
2002 d'une revue en ligne, Surveillance \& Society.

13 C'est également l'une des conclusions auxquelles parvient Michaelis Lianos dans sa thèse de doctorat (1996) : il mobilise l'image de la société péri-optique pour suggérer qu'il existerait de la surveillance à distance à tous les échelons de la société.

14 C'est l'une des conclusions auxquelles parvient par exemple une étude britannique des réseaux locaux d'action publique en matière de sécurité (Fussey, 2004).

15 Voir notamment l'ouvrage dirigé par Stéphane Leman-Langlois (2008), qui comprend à la fois des chapitres où des auteurs « patentés » des surveillance studies développent leurs analyses et une introduction, une conclusion et d'autres chapitres qui discutent vigoureusement ce courant.

16 Le Gouvernement de Tony Blair a élaboré 21 «boîtes à outils » à disposition des partenariats locaux. La vidéosurveillance est présente dans neuf d'entre elles et notamment préconisée dans la lutte contre les désordres, le sentiment d'insécurité et la victimation répétée (les principaux problèmes identifiés au niveau local).

17 Entre 1994 et 1996, sous le gouvernement de John Major, le Home Office finance à 50\% l'installation de dispositifs via des appels à projets à hauteur de 37 millions de livres. L'effort de financement se poursuit sous le gouvernement de Tony Blair, arrivé au pouvoir en 1997 : 38 millions de livres servent au financement de 585 dispositifs entre 1996 et 1998 puis 170 millions de livres entre 1999 et 2001 (Chiffres du Home Office Policing and Reducing Crime Unit).

18 E. Heilmann et M.-N. Mornet (2001) y voient d'ailleurs une spécificité propre au cas britannique.

19 Pour une analyse de ces codes de bonne conduite, voir Norris et Armstrong, 1999b. Pour un examen des limites des pratiques des collectivités locales britanniques, voir Buclos, Sarno, 1996.

20 Commission Nationale Informatique et Liberté.

21 La loi d'orientation et de programmation pour la sécurité (LOPS) de 1995, qui comporte un volet relatif à la vidéosurveillance, a suscité un certain nombre d'analyses sur le mode de régulation mis en place (Favoreu, 1995 ; Pellet, 1995 ; Forest, 1999) et les modalités d'application des textes (Darras, Deharbe, 1996 ; Ocqueteau, Heilmann, 1997 ; Ocqueteau, 2001).

22 Sans compter que l'argument s'appuie parfois sur des enquêtes à la méthodologie douteuse : Jason Ditton (1998) a ainsi montré que le soutien du public à la vidéo variait de 35 points selon l'agencement du questionnaire.

23 Auxquels il faudrait ajouter la disponibilité de la technique et l'existence d'un marché de la vidéosurveillance (Graham, 1998 ; Ocqueteau, 2004).

24 Il convient de vérifier la validité de leur chèque (absence d'interdit bancaire) tout en prévenant les possibilités de scandale. S'il s'avère que le chèque est effectivement non valable, la vidéosurveillance permet de garder une trace de la scène du scandale perpétré par le "mauvais payeur".

25 À Lyon, par exemple, si les Verts refusent initialement de jouer la solidarité au sein de la " gauche plurielle », ils n'en sont pas moins enrôlés dans le dispositif via le collège d'éthique qui joue ici le rôle d'un dispositif d'intéressement. Dans ces conditions, il est moins étonnant de voir, à partir de 2005, les Verts lyonnais voter pour certains projets locaux d'extension de la vidéosurveillance.

\section{Pour citer cet article}

Référence électronique

Laurence Dumoulin, Séverine Germain et Anne-Cécile Douillet, « Une petite entreprise qui ne connaît pas la crise », Champ pénal / Penal field, nouvelle revue internationale de criminologie [En ligne] , Vol. VII | 2010 , mis en ligne le 09 novembre 2010, Consulté le 18 mars 2011. URL : http://champpenal.revues.org/7931

\section{Auteurs}


Laurence.Dumoulin@isp.ens-cachan.fr

\section{Séverine Germain}

ATER à l'université de Rouen, chercheuse associée à PACTE (UMR 5194, CNRS/IEP de Grenoble/Université Pierre Mendès-France/Université Joseph Fourier).

severine.germain@gmail.com

\section{Anne-Cécile Douillet}

Maître de conférences à l'université de Franche-Comté, chercheuse associée à PACTE (UMR 5194, CNRS/IEP de Grenoble/Université Pierre Mendès-France/Université Joseph Fourier).

anne-cecile.douillet@univ-fcomte.fr

\section{Droits d'auteur}

(C) Champ pénal 